Parcours de recherche en SIC: littérature scientifique, méthodes et terrain

\title{
Les SIC face à la nécessité des références théoriques classiques
}

ICS Studies versus the Necessity of Traditional References

\section{Zeineb Touati}

\section{(2) OpenEdition}

Journals

Édition électronique

URL : http://journals.openedition.org/edc/858

DOI : $10.4000 /$ edc.858

ISSN : 2101-0366

Éditeur

Université Lille-3

Édition imprimée

Date de publication : 1 avril 2009

Pagination : 19-32

ISBN : 978-2-917562-01-7

ISSN : $1270-6841$

Référence électronique

Zeineb Touati, «Les SIC face à la nécessité des références théoriques classiques », Études de communication [En ligne], 32 | 2009, mis en ligne le 01 juin 2011, consulté le 19 avril 2019. URL : http:// journals.openedition.org/edc/858; DOI : 10.4000/edc.858

Ce document a été généré automatiquement le 19 avril 2019

(c) Tous droits réservés 


\title{
Les SIC face à la nécessité des références théoriques classiques
}

\author{
ICS Studies versus the Necessity of Traditional References
}

Zeineb Touati

1 Les SIC disposent de méthodes et de concepts permettant d'étudier différents types d'objets et de phénomènes y compris les plus actuels et les plus complexes. Certains objets d'études combinés à des terrains spécifiques suggèrent parfois de sortir du cadre théorique établi par les SIC et de faire appel à des références théoriques classiques jugées parfois comme très anciennes - ou encore à des références relevant d'autres disciplines éloignées des SIC ; ceci tout en travaillant sur une problématique relevant des SIC et en adoptant une approche communicationnelle précise et bien établie. C'était le cas de notre recherche de thèse intitulée Communication et relations symboliques : les représentations sociales des femmes entre les médias, la politique, la culture et l'Islam, soutenue à l'Université Stendhal en Octobre 2006. Dans cette thèse, nous avons analysé le rôle des moyens de communication de masse (télévision, cinéma et presse écrite) dans la construction, l'évolution et la disparition des représentations sociales des femmes. Nous avons traité du rôle des médias, des institutions (Etat, famille, école et instances religieuses) et des processus symboliques dans la construction et l'évolution des représentations sociales des femmes dans la société tunisienne. Ceci afin de comprendre l'interdépendance des processus de représentations (valeurs, idées, opinions) et de communication (médias, discours, relations publiques).

2 Tout au long, de ce travail de recherche, notamment lors de quelques réunions de doctorants comme pendant la soutenance de thèse, nos choix de lecture et de références théoriques ont interpellé et ont suscité débat et questions. C'est en réponse à certaines de ces questions et sur la base des lectures et des choix théoriques effectués lors de la thèse que nous proposons dans cette contribution d'engager une réflexion sur les différents positionnements théoriques adoptés en SIC pour analyser la production des représentations sociales. Nous présenterons, dans une première partie, la nécessité de s'ouvrir à d'autres disciplines, y puiser des lectures et des références théoriques ainsi que la nécessité de se référer aux auteurs classiques tels que P. Bourdieu ou C. Castoriadis. 
Nous y expliquerons les raisons qui nous ont amenée à combiner des écrits et des concepts "classiques" qui paraissent pour certains hétérogènes, que plusieurs considèrent comme très anciens et qu'une majorité juge éloignés des SIC. Dans une seconde partie, nous mettrons en avant les difficultés qu'entraînent de tels choix théoriques et la nécessité de les justifier.

\section{Classiques et interdisciplinarité indispensables à l'étude des représentations sociales}

\section{L'élargissement à la sociologie de la connaissance}

3 Dès l'élaboration de la problématique, nous avons compris que cette dernière s'intégrait parfaitement dans le cadre théorique de la sociologie de la connaissance qui s'intéresse à la construction sociale de la réalité bien au-delà de l'élaboration théorique par des spécialistes du travail intellectuel. Elle consiste en l'analyse sociologique de la réalité de la vie quotidienne, précisément de la connaissance commune qui guide les conduites personnelles dans la vie quotidienne. On sort, ici, du cadre de l'analyse des idées et des pensées pour l'élargir à celui des actions ou des activités objectives toujours sous-tendues par des significations subjectives qui participent de cette réalité de la vie quotidienne. Notre choix du cadre théorique de la sociologie de la connaissance est déterminé par le double intérêt qu'elle porte à l'insertion sociale des représentations individuelles et à leur caractère idéologique et pratique mélangé. En effet, la plupart des femmes (et des hommes) n'a pas une conscience claire et s'interroge encore moins de façon philosophique ou scientifique sur son statut social vécu comme donné et établi. Ceci se voit en particulier dans le cas de la culture traditionnelle arabo-musulmane où la place de la femme est fortement déterminée par sa socialisation et son éducation dès l'enfance et ce depuis de nombreuses générations. La connaissance pratique de ce qu'une femme doit faire ou ne pas faire, doit penser ou ne pas penser, doit dire ou ne doit pas dire est naturalisée à la fois par la socialisation primaire et par l'emprise des valeurs sociales et des représentations religieuses sur toute la vie des femmes. Néanmoins, celles-ci sont des individus singuliers qui ont leur pensée propre et qui réagissent diversement à la situation qui leur est faite et aux idées qui leur sont inculquées. Dans ces réactions où se manifestent des frustrations d'ordre psychique ou sexuel par exemple, des résistances (refus d'un mari, refus de l'enfermement), voire des rebellions (refus d'assumer le devoir conjugal d'ordre sexuel, prendre un amant), les transformations du contexte social et la circulation de représentations alternatives exercent un rôle important. La compréhension de cette circulation entre connaissances pratiques intériorisées et transformations des représentations sociales était indispensable à notre problématique ; d'où l'intérêt et l'apport de la sociologie de la connaissance. Par ailleurs, le lien entre la sociologie de la connaissance et les représentations sociales est clair à partir du moment où l'on admet qu'en tant que mode de connaissance, la représentation suppose la capacité à traduire le réel dans un cadre social de vie quotidienne et sous la forme de pensées qui s'y accordent. Il s'agit d'une reconstitution du réel qui n'est, certes pas, neutre ni impartiale, dans laquelle l'individu qui y procède croit être fidèle à la réalité et n'avoir déformé aucun de ses aspects.

4 Le cadre théorique de la sociologie de la connaissance nous a amenée à découvrir des auteurs et des approches auxquelles nous n'avions pas pensé lors du choix du sujet et de 
l'élaboration de la problématique. Certaines de ces lectures auxquelles nous nous sommes référée dans la thèse ne faisaient pas l'unanimité dans le domaine des SIC. Parmi ces références, nous citons $L a$ construction sociale de la réalité des sociologues nord-américains (Berger, Luckmann, 1986), nous y avons puisé ce qu'ils appellent un «stock social commun » et définissent comme un ensemble objectivé de connaissances ou de représentations communément admises qui se constitue au fur et à mesure de l'accumulation « sélective " des expériences personnelles et historiques accumulées dans une culture sociale. Socialement produit et partagé par les membres d'une même société ou communauté, ce stock résulte des expériences individuelles et collectives ainsi que des événements historiques, des faits sociaux objectivés et accumulés. Il s'agit d'une action sur le long terme qui dépasse la vie d'une ou de plusieurs générations, c'est l'histoire entière d'une communauté ou d'un groupe social qui entre en jeu. Cette indication est importante pour notre sujet. En effet, il y a bien la rencontre entre une très longue histoire objectivée dans la culture arabo-musulmane et les pratiques qu'elle engendre, avec ce qu'entraîne variablement le statut de la femme tunisienne.

5 Le concept de «Stock social » s'adapte parfaitement à la réalité de l'imaginaire arabomusulman, socle et origine des principales représentations, pratiques et valeurs sociales dans la société tunisienne. Même avec le recul pris depuis la soutenance, nous sommes convaincus que sans le recours à la notion de "Stock social» et aux niveaux de légitimation développés par $\mathrm{P}$. Berger et $\mathrm{T}$. Luckmann, nous n'aurions pas pu appréhender avec autant de précision, ni approcher d'aussi près les composantes et les niveaux de l'imaginaire arabo-musulman et par conséquent les modes de légitimation des représentations sociales répertoriées.

6 L'ancrage dans la sociologie de la connaissance est contestable en SIC pour certains des chercheurs que nous avons côtoyés. Certains considèrent la sociologie de la connaissance comme très éloignée des SIC, d'autres la jugent comme une discipline très récente et fortement influencée par la sociologie nord-américaine, donc à manier avec précaution. Toutefois, cet ancrage s'est avéré indispensable pour répondre à notre problématique et traiter spécifiquement du terrain tunisien, du quotidien, des pratiques et des valeurs gérant la vie des femmes et les représentations faites des femmes tunisiennes.

\section{Les représentations sociales : un concept transversal en mouvement}

7 Un objectif tel que celui que nous nous sommes fixés, à savoir analyser les modes de construction et d'évolution des représentations sociales des femmes et étudier les rôles des médias, de la politique, de la religion et de la culture dans ces processus, nécessite la mobilisation d'un certain nombre de théories relevant aussi bien de la sociologie, de la psychosociologie que des SIC elles-mêmes. Nous avons, en effet, rapidement saisi que s'intéresser à cet objet, c'est accepter de questionner un objet aux frontières diffuses, socialement construit dans l'interaction médiatique, culturelle, politique et religieuse, interactions qui produisent autant d'aller-retour producteurs d'une image sans cesse en mouvement. Pour montrer le caractère instable et évolutif des représentations et de leurs fondements, nous avons dû rassembler des travaux développés par des chercheurs d'horizons différents et des concepts considérés habituellement comme hétérogènes. Ainsi, par exemple, nous avons associé des notions telles que : la domination et la violence symbolique développées par P. Bourdieu (sociologie), la notion de représentations 
sociales de S. Moscovici (psychosociologie) et celle des significations imaginaires sociales développée par C. Castoriadis (philosophie). C'est également dans cette perspective que nous avons fait appel aux écrits de P. Berger et T. Luckmann (sociologie de la connaissance). Notre approche, en articulant ces différents concepts théoriques nous permet d'accéder au plus près de l'objet étudié pour l'appréhender dans toute son épaisseur et sa complexité.

En effet, en plus du cadre théorique général qui nous a orientée vers des lectures éloignées des SIC et qui nous a amenée à affirmer un positionnement théorique clair en sociologie de la connaissance, le concept même de représentations sociales a influencé l'orientation bibliographique et théorique de la recherche. Nous nous sommes rapidement rendus compte que le concept de représentations sociales que nous croyions pouvoir aborder du point de vue des sciences de l'information et de la communication, s'est avéré difficile à saisir par sa transversalité et son caractère interdisciplinaire et pluridisciplinaire. Dénomination « carrefour » ou notion «valise », elle est présentée par les psychologues sociaux comme une «jonction entre les domaines de l'individuel et du social, articulation des approches cognitives et sociales, pluridisciplinaire enfin par l'intérêt qu'y portent historiens des mentalités, linguistes, sociologues, anthropologues et psychologues sociaux" (Bonardi, Roussiau, 1999, p. 7).

9 Cette pluridisciplinarité du concept est également mise en avant par W. Doise qui affirme que «le carrefour auquel se trouve la notion de représentations sociales est particulièrement dense, les voies qui y débouchent sont multiples et il n'y a aucune carte qui en donne les coordonnées communes. En effet, psychanalystes, cliniciens, psychologues, psychosociologues, sociologues, historiens peuvent converger vers ce carrefour tout en croyant se déplacer dans des espaces différents de sorte qu'ils ne devraient jamais se rencontrer» (Doise, 2002, p. 82). Chacune de ces disciplines a récupéré le concept à sa façon et l'a traité selon la méthode ou l'approche qui lui convient le mieux mais elles ont toutes gardé des liens entre-elles qui sont essentiellement dus à l'héritage philosophique commun présentant le cadre général des représentations sociales. Ces liens directs ou indirects d'explication et même de justification nous ont paru indispensables à une théorisation des représentations sociales et sont à la base de toute tentative de présentation et de définition.

Comprendre ce concept nécessite donc de tenir compte de l'apport de chacune des disciplines qui l'ont adopté comme thème central et du caractère complémentaire ou bien contradictoire des définitions qu'elles en offrent. C'est ce constat qui explique et qui justifie notre recours à beaucoup d'écrits en psychologie et en psychologie sociale, en plus de ceux relevant de la sociologie. C'est d'ailleurs, grâce à ces lectures différentes et variées, que nous avons pu saisir le sens de ce concept, en dégager les caractéristiques fondamentales et que nous avons pu en extraire la construction d'un point de vue cohérent et opératoire adapté à la nature de notre terrain et qui nous a permis d'appréhender la culture arabo-musulmane et l'identité féminine dans leurs complexités et dans leurs interactions.

11 La première discipline à avoir envisagé le lien entre représentations sociales et médias a été la psychologie sociale. D'où notre intérêt pour des écrits relevant de cette discipline. Nous ne pouvions pas ignorer l'apport considérable du psychologue social S. Moscovici qui a été le premier à établir le lien entre les médias de masse et l'évolution des représentations sociales. Tout en partant de l'héritage durkheimien, S. Moscovici passe des représentations collectives aux représentations sociales qu'il envisage comme 
dynamiques à l'instar des sociétés contemporaines (Moscovici, 1961). La définition de S. Moscovici a été développée et enrichie par d'autres tels que P. Moliner, P. Rateau, C. Bonardi, etc. qui ont également traité la question des relations entre médias et représentations sociales du point de vue de la psychologie sociale. Nous avons choisi de puiser dans certaines de leurs définitions qui complétaient celle de S. Moscovici, jugée très ancienne parce que datant de 1961. Nous nous sommes contentée de nous référer aux définitions données par les auteurs cités précédemment sans pour autant nous référer à leurs résultats, méthodologies ou ancrages théoriques très éloignés des SIC et qui représentaient par conséquent un risque considérable pour l'ancrage de notre recherche en SIC.

12 Chez P. Berger et T. Luckhmann, nous avons emprunté la conception des univers symboliques. Construction cognitive émanant des processus de réflexion subjective et des structures mentales visant à légitimer un ordre et des institutions socialement établis. Ceci en leur donnant une signification et un sens et assurant, de la sorte, la maittrise de l'environnement, purement symbolique à la base, objectivé ainsi que socialement produit et partagé. L'univers symbolique correspond à la conception des représentations sociales données par les psychosociologues. Nous avons rapproché la conception des univers symboliques avec celle de $\mathrm{C}$. Castoriadis bien qu'il inclut les représentations sociales dans l'ensemble plus englobant de l'imaginaire social et historique d'une société donnée. Il parle de « significations imaginaires sociales " qui « ne sont évidemment pas ce que les individus se représentent, consciemment ou inconsciemment, ou ce qu'il pensent. Elles sont ce moyennant et à partir de quoi les individus sont formés comme individus sociaux, pouvant participer au faire et au représenter/dire social, pouvant représenter, agir et penser de manière compatible, cohérente, convergente même si elle est conflictuelle.» (Castoriadis, 1975, p. 528). Ces significations imaginaires sociales diffèrent évidemment d'une société à une autre et forment, selon le philosophe et psychanalyste, une sorte de «magma» dans et par lequel la société prend sens, institue et s'institue. Le rôle principal de ces significations est d'apporter des réponses aux questions individuelles et sociales cruciales. Ce rôle est assuré chez $\mathrm{P}$. Berger et $T$. Luckhmann par les "schémas typificatoires" qui gèrent les interactions sociales et qui vont - en grande partie - nous dicter les comportements à suivre et les attitudes à adopter dans telle ou telle situation. C'est dans ces schémas typificatoires que les femmes tunisiennes puisent inconsciemment des manières de penser et des façons d'agir sans pour la majorité d'entre-elles se poser la question de leur validité ou de leur objectivité. Dans ces schémas, elles trouvent des règles de conduite leur imposant le respect et la pudeur lors de l'interaction avec le père, ils leur indiquent la nécessité de la soumission face à la belle-mère ou encore ils leur inculquent des façons de tenir le corps, de serrer les jambes et pour certaines de rougir et de baisser le regard en présence d'un étranger. Nous retrouvons une idée similaire chez P. Bourdieu qui parle d'une «logique pratique » totalement différente de la logique intellectuelle des «savants». Selon lui cette logique pratique fonctionne sur la base de schèmes classificatoires pré-réflexifs composant les "habitus». Bien que dans un système théorique différent, il y a chez $\mathrm{P}$. Bourdieu l'idée que ces schèmes représentationnels sont liés à des groupes sociaux spécifiques auxquels il donne le nom de « champs » sociaux.

Les schémas typificatoires se rapprochent étroitement de la notion d'habitus agissant dans un champ social donné qui désigne l'ensemble des dispositions acquises, sous forme de « catégories » de perceptions, de pensées et d'actions données à l'individu dans et par un contexte social donné lui permettant de réguler, inconsciemment, ses décisions et ses 
comportements. Le lien entre habitus et représentations sociales apparaît évident si l'on envisage les représentations comme forme de connaissance et d'interprétation communes de la réalité. Il nous faut, toutefois, préciser que contrairement aux psychologues sociaux cités précédemment (Moscovici, Moliner, Jodelet, Roussiau, Rateau, Bonardi, Doise) mais en accord avec des théoriciens constructivistes (Berger et Luckmann), l'inspiration structuraliste de P. Bourdieu présente les représentations (quoique n'employant jamais ce concept) que possèdent ou dont disposent les individus comme liées et fortement dictées et/ou déterminées par la position sociale de ces individus dans un champ social donné. Pour P. Bourdieu et pour P. Berger et T. Luckmann, les rapports sociaux sont l'élément ou l'instance qui, détermine et explique, l'apparition et l'évolution de telle ou telle autre représentation et permet d'expliquer et d'interpréter les éventuelles relations entre représentations sociales. Le modèle de l'habitus envisage donc les représentations comme directement et intimement déterminées par certaines conditions sociales dont la plus importante serait l'appartenance à telle ou telle classe sociale en même temps qu'à tel ou tel champ social, comme l'attestent ses travaux sur l'école, les carrières professionnelles, le goût, etc. Les représentations ne sont alors que l'expression ou la traduction des habitus émanant de champs sociaux précis. Les pratiques, les échanges et les interactions ne sont que «le produit de dispositions qui, étant l'intériorisation des mêmes structures objectives, sont objectivement concentrées, (...) les pratiques des membres d'un même groupe (...) sont dotées d'un sens objectif à la fois unitaire et systématique, transcendant aux intentions subjectives et aux projets conscients, individuels ou collectifs.» (Bourdieu, 1972, p. 183). Cette conception théorique s'exprime de façon immédiate lorsque l'on compare la position et les représentations des femmes tunisiennes selon qu'elles vivent dans un milieu rural traditionnel ou dans une famille instruite dans une grande ville.

La combinaison de ces notions relevant de différentes disciplines nous a permis de construire notre propre définition des représentations sociales des femmes, une définition opératoire et pertinente pour permettre de répondre à notre problématique et de correspondre aux limites imposées par l'objet et le terrain. Mais elle pose certains problèmes que nous présentons dans la partie suivante.

\section{Les problèmes posés}

Les choix d'auteurs et de lectures que nous avons faits et qui nous ont été imposés par la nature du terrain et l'étendue des concepts clefs sont facilement contestables en SIC et c'est pour cette raison qu'ils ont fait l'objet de plusieurs reproches et questions en raison de leurs conséquences d'ordre épistémologique et méthodologique. Questions et reproches formulées par des spécialistes des SIC lors de réunions de laboratoire et surtout par les membres du jury pendant la soutenance de la thèse.

Parmi les problèmes soulevés par nos choix de lectures, la présence dans notre bibliographie de plusieurs ouvrages considérés comme très anciens puisque datant des années 60 et 70 (Moscovici, 1961, Castoriadis, 1975 et Bourdieu, 1972, 1979). Il est vrai que ces ouvrages datent de plusieurs décennies mais ceci n'implique pas qu'ils soient dépassés ou sans intérêt. Les écrits de C. Castoriadis, de P. Bourdieu et de bien d'autres sont encore aujourd'hui des textes de référence dans plusieurs domaines. Nous sommes convaincus que nous n'aurions pas pu traiter notre sujet, ni répondre à notre problématique sans faire appel aux concepts de «Domination masculine » et de «Violence symbolique » mis en 
avant par P. Bourdieu dans les années 80. Ces concepts pourraient paraître dépassés lorsqu'on étudie les rapports sociaux de sexe, les représentations des femmes ou encore les images médiatiques des femmes dans les sociétés européennes ou américaine. Il n'en est pas de même pour la société patriarcale tunisienne véhiculant un lourd héritage arabo-musulman, avec des rapports sociaux, des pratiques et des valeurs fortement marqués par la supériorité masculine. Le recours à des concepts plus récents et à la mode en Europe comme celui du "Genre » n'était nullement adapté au terrain tunisien. Les concepts fondamentaux, en dépit de leur ancienneté, sont, en effet, indispensables pour cerner certains objets d'étude et éclairer des problématiques complexes.

Se pose également le problème des auteurs et concepts éloignés des SIC, qui pour certaines personnes remettrait en question l'approche communicationnelle et l'ancrage de la recherche en SIC. Consciente des ancrages et des traditions épistémologiques bien établis des disciplines mobilisées et de leurs différences avec les SIC, nous avons clairement explicité notre approche communicationnelle fondée sur une problématique tentant de prouver et d'expliquer les interactions entre médias et processus symboliques et traitant de contenus et de processus médiatiques et de modes de réception. Le recours à des concepts éloignés des SIC ne représente pas en soi une négation de l'ancrage en SIC. La nature du sujet combinée à la nature interdisciplinaire des SIC elles-mêmes, impose l'élargissement à d'autres disciplines plus anciennes ou ayant traité des représentations sociales sous d'autres angles. La combinaison de plusieurs auteurs (Moscovici, Castoriadis et Bourdieu) appartenant à diverses disciplines et qui ne se sont jamais mutuellement cités était risqué mais se justifiait par la nécessité de construire une définition la plus opératoire possible des représentations sociales. Chose qui nous a amené à combiner plusieurs conceptions, tout en respectant leurs différences et les nuances existant entre elles.

Le choix de ces lectures et l'appropriation de conceptions relevant d'autres disciplines, pose également le problème de l'ancrage épistémologique. Tout en respectant les approches des auteurs cités et leurs traditions épistémologiques, nous nous sommes cantonnée à une méthodologie et un ancrage en SIC. C'est ainsi, par exemple, que nous avons choisi de ne puiser chez les psychosociologues que leurs définitions des représentations sociales et de laisser de coté leurs méthodes, basées d'une part, sur la mobilisation d'échantillons considérables dépassant nos capacités, et d'autre part sur une séparation, déterminante en psychosociologie, entre noyau central et périphérie mais qui n'était d'aucun intérêt pour notre travail.

\section{Conclusion}

Les SIC ont produit des concepts propres à la discipline ainsi que les outils méthodologiques permettant de les étudier, ce qui a permis aux chercheurs en Info/Com de traiter plusieurs objets et de questionner nombre de phénomènes parmi les plus complexes. Ceci n'empêche, toutefois, pas les chercheurs en SIC de recourir dans certains cas à des concepts forgés et développés dans d'autres disciplines. Il s'agit ici d'une spécificité avantageuse des SIC qui marque leur ouverture puisqu'elles permettent aux chercheurs de la discipline de formuler des problématiques complexes sans les enfermer a priori dans des modes de pensée ou les enfermer dans des moules contraignants en leur imposant des concepts à utiliser et des méthodes à mobiliser. Les SIC offrent aux 
chercheurs une certaine marge de manœuvre lorsqu'ils traitent de certaines problématiques telle que celle que nous avons étudiée.

La complexité de la problématique alliant l'étude des interactions sociales, des processus symboliques et des discours médiatiques et la nature du terrain de recherche, nous ont amenée à faire des choix théoriques et méthodologiques et à "bricoler " une définition des représentations sociales afin de construire une partie théorique nous permettant le plus possible d'approcher et d'apprivoiser notre objet de recherche. Les choix de lecture sont directement dictés et initiés par la nature du sujet et de l'objet mais également par la nature interdisciplinaire des SIC. Le recours aux classiques et aux auteurs fondamentaux, qui peuvent paraître anciens ou dépassés, est encore indispensable et peut se justifier par la nécessité de maîtriser les concepts, de délimiter les courants de pensée et de cerner les objets de recherche. L'ouverture à d'autres disciplines, avec des traditions épistémologiques établies, est un exercice périlleux mais nécessaire dans plusieurs types de recherches notamment celles qui mobilisent des concepts rarement traités en SIC.

\section{BIBLIOGRAPHIE}

Berger, P. et Luckmann, T., (1986), La construction sociale de la réalité, A. Colin, Paris, 288 p.

Bonardi, C. et Roussiau, N., (1999), Les représentations sociales, Paris, Dunod, 124 p.

Bourdieu, P., (1972), Esquisse d'une théorie de la pratique, Genève, Droz, 269 p.

Bourdieu, P., (1998), La domination masculine, Paris, Minuit, 142 p.

Castoriadis, C., (1975), L'institution imaginaire de la société, Paris, Seuil, 497 p.

Doise, W., (2002), L'étude des représentations sociales, sous la direction de W. Doise et de A. Palmonari, Paris, Delachaux et Niestlé, 207 p.

Moliner, P., (1996), Images et représentations sociales. De la théorie des représentations à la l'étude des images sociales, Grenoble, PUG, 275 p.

Moscovici, S., (1961), La psychanalyse, son image, son public, Paris, PUF, 651 p.

\section{NOTES}

1. Pour plus de précisons sur ce point voir la conception de C. Herzlich in P. Moliner, Images et représentations sociales. De la théorie des représentations à la l'étude des images sociales, Grenoble, PUG, 1996, p. 24 et suivantes.

2. Il est le premier à mettre l'accent sur le rôle des médias et des moyens de communication dans la fabrication et la pérennisation des représentations sociales. Dans son ouvrage La psychanalyse : son image, son public, paru en 1961, il se livre à l'étude des différentes représentations inspirées par la psychanalyse dans les journaux américains. S'intéressant à la nature de différents supports journalistiques et à leurs impacts dans la diffusion des connaissances sur l'objet " psychanalyse ", il démontre que les représentations de la psychanalyse se modifient quand elles sont actualisées 
sous différents systèmes de communication à savoir: la diffusion, la propagation et la propagande. Il démontre que ces modes de communication qui relèvent des médias de masse et donc de l'espace public, agissent en premier lieu sur l'élaboration de la représentation d'un objet, mais peuvent par la suite, générer des transformations plus ou moins profondes de cette même représentation.

3. Le magma est pour C. Castoriadis «le mode d'organisation d'une diversité non ensemblisable, exemplifié par le social, par l'imaginaire ou par l'inconscient ». C. Castoriadis, ibid, p. 273.

\section{RÉSUMÉS}

Cette contribution se propose d'engager une réflexion sur les différents positionnements théoriques et épistémologiques adoptés en SIC pour analyser la production des représentations sociales et la nécessité de s'ouvrir à d'autres disciplines. Ceci en nous référons aux lectures et aux choix théoriques effectués lors de notre travail de thèse qui a nécessité la mobilisation d'un certain nombre de théories relevant aussi bien de la sociologie, de la psychosociologie que des SIC elles-mêmes. Nous expliquons les raisons qui nous ont amené à combiner des écrits et des concepts «classiques" qui paraissent pour certains hétérogènes, que plusieurs considèrent comme très anciens et qu'une majorité jugent éloignés des SIC. Nous témoignons des résistances auxquelles nous avons dû faire face et comment nous avons justifié et argumenté nos références théoriques. Nous montrons, en parallèle, les limites et les enjeux épistémologiques d'une telle approche dans le cadre des études en SIC et les biais que peut entraîner une combinaison simultanée de plusieurs concepts empruntés à différentes disciplines aux traditions épistémologiques bien établies.

This paper proposes a study of various theoretical and epistemological aspects used in SIC in order to analyze the production of social representations and the necessity of opening the SIC domain over other disciplines. The reasons underlying the fact that we focus particularly on our readings and on the theoretical choices carried out during our PhD thesis, are presented. In fact, our $\mathrm{PhD}$ thesis work had required the mobilization of a number of theories associated to sociology, social psychology as well as to SIC field itself. This paper proposes to combine writings and "traditional" concepts that seem to be very old and which are considered by many researches as out of SIC subject. Furthermore, the limits and the epistemological stakes of such an approach are proposed in the framework of the SIC studies.

\section{INDEX}

Keywords : social representation, interdisciplinarity, information science, communication science, knowledge sociology

Mots-clés : représentation sociale, interdisciplinarité, sciences de l'information et de la communication, sociologie de la connaissance 


\section{AUTEUR}

\section{ZEINEB TOUATI}

Zeineb Touati est docteure en Sciences de l'Information et de la Communication (2006) de l'Université Stendhal (Grenoble 3). Elle est également enseignante au département Information/ Communication de l'Université du Havre. Membre du laboratoire CIRTAI (UMR IDEES). Elle est titulaire d'un Master en Sciences Politiques de l'IEP de Grenoble et d'un DEA en Sciences de l'Information et de la Communication de l'Université Stendhal (Grenoble 3). 